\title{
ANALYSIS OF SERVICEABILITY OF FULL-ENGAGEMENT GEAR TRAINS FOR REDUCTION GEARS IN SCROLL CENTRIFUGES
}

\author{
A. Ya. Krasilnikov ${ }^{1}$ and K. Yu. Muravyov ${ }^{2}$
}

An experimental unit for confirming the serviceability of gear trains with different tooth geometry, which are employed in the reduction gears of scroll centrifuges, is examined. Results are presented for comparative analysis of two gear pairs with different tooth geometry.

In a settling scroll centrifuge, a separate precipitate is transported due to the difference in the rotational speed of the rotor and scroll, which may range from 10 to $60 \mathrm{rpm}$.

Figure 1 shows one of the kinematic diagrams of a centrifuge, which provides for different turning speeds of the rotor and scroll. The rotation is transferred from one drive via belt transmissions onto carrier $H$ and gear 4 , whereupon the gear ratios of the belt transmissions differ. The carrier and gear are members of a two-step reducer in which cylindrical internally meshed gears with small differences in number of teeth (teeth 1-4) are used.

The kinematics of this reduction gear is discussed in [1].

The gear ratio of the reduction gear when member 4 is at rest $\left(u_{H 1}^{(4)}\right)$ is determined from the formula [2]:

$$
u_{H 1}^{(4)}=\frac{1}{1-\frac{z_{1} z_{4}}{z_{2} z_{3}}},
$$

where $z_{1}-z_{4}$ are, respectively, the numbers of teeth on each member.

As is apparent from formula (1), $u_{H 1}^{(4)}$ will be greatest, if $z_{1} z_{4} / z_{2} z_{3} \rightarrow 1$, i.e., when $z_{2}-z_{1}=1, z_{4}-z_{3}=1$, and $z_{2} / z_{1} \neq z_{4} / z_{3}$.

Gear pairs with evolute internal engagement are normally used in the reduction gears of scroll centrifuges. When the difference in the numbers of gear teeth is small (from 1 to 3 ), this engagement will be completely polar: tooth contact occurs not at the pitch point, but at a point located between the pitch point and the axis of rotation of the drive gear (i.e., prior to the pole). Use of this engagement makes it possible to build compact gear transmissions with a high gear ratio, and also differential-planetary transmissions.

Basic problems preventing the use of these transmissions are: analysis of their geometry is difficult; and, since teeth of reduced height are used in the transmissions, it is necessary to cut them with a nonstandard tool.

To find a solution to problems involving the use of fully polar gearing, we fabricated a device for an experimental reduction gear - the simplest single-step cylindrical reduction gear with replaceable gears 3 and 4 (Fig. 2). Two pairs of gears

\footnotetext{
${ }^{1}$ Yeltsin Ural Federal University (UrFU), Yekaterinburg, Russia.

${ }^{2}$ Sverdlovskii Research and Design Institute of Chemical Machine Building (SverdNIIkhimmash), Yekaterinburg, Russia.
} 


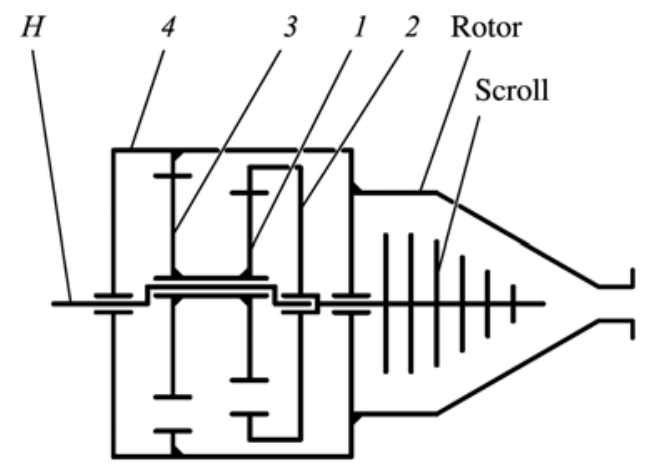

Fig. 1. Simplified kinematic diagram of scroll centrifuge: 1,3 ) gears with external teeth; 2) gear with internal teeth; 4) gear with internal teeth, which is connected to housing of reduction gear; $H$ - carrier.

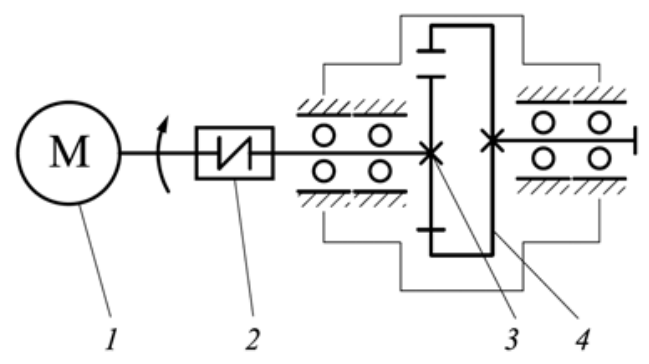

Fig. 2. Kinematic diagram of experimental device: 1) electric motor; 2) clutch; 3) drive gear with external teeth; 4) driven gear with internal teeth.

with teeth of reduced height (tooth-point height factor $h_{a}^{*}=0.8$ ) were tested on the device. The teeth of the first gear pair were cut by a nonstandard tool (cutter with cut tooth points), and the teeth of the second gear pair by a standard tool. Comparative serviceability analysis of the gearing was performed by sequential run-in of each pair of gears on the experimental device (see Fig. 2).

The geometry of the internal engagement was calculated by the procedure outlined in [3]. The following problems arose in analyzing complete engagement based on this procedure: it is necessary to calculate the values of the inverse-involute function when determining the inter-axial distance; and, it is impossible to determine analytically the interference in the engagement.

Problems encountered in determining the interference in the engagement are discussed in [4] and [5].

The values of the inverse-involute function were calculated in accordance with the procedure outlined in [6]. For a known $\varphi=\operatorname{inv} \alpha$, the preliminary value of the angle $\alpha_{0}$ is determined from the formulas:

$$
\alpha_{0}=1.441 \varphi^{1 / 3}-0.374 \varphi
$$

when $\varphi<1.8$;

$$
\alpha_{0}=1.308997+0.0717968 \zeta-0.0206191 \zeta^{2}+0.006517008 \zeta^{3}-0.001238501 \zeta^{4},
$$

when $1.8<\varphi<5$; and 

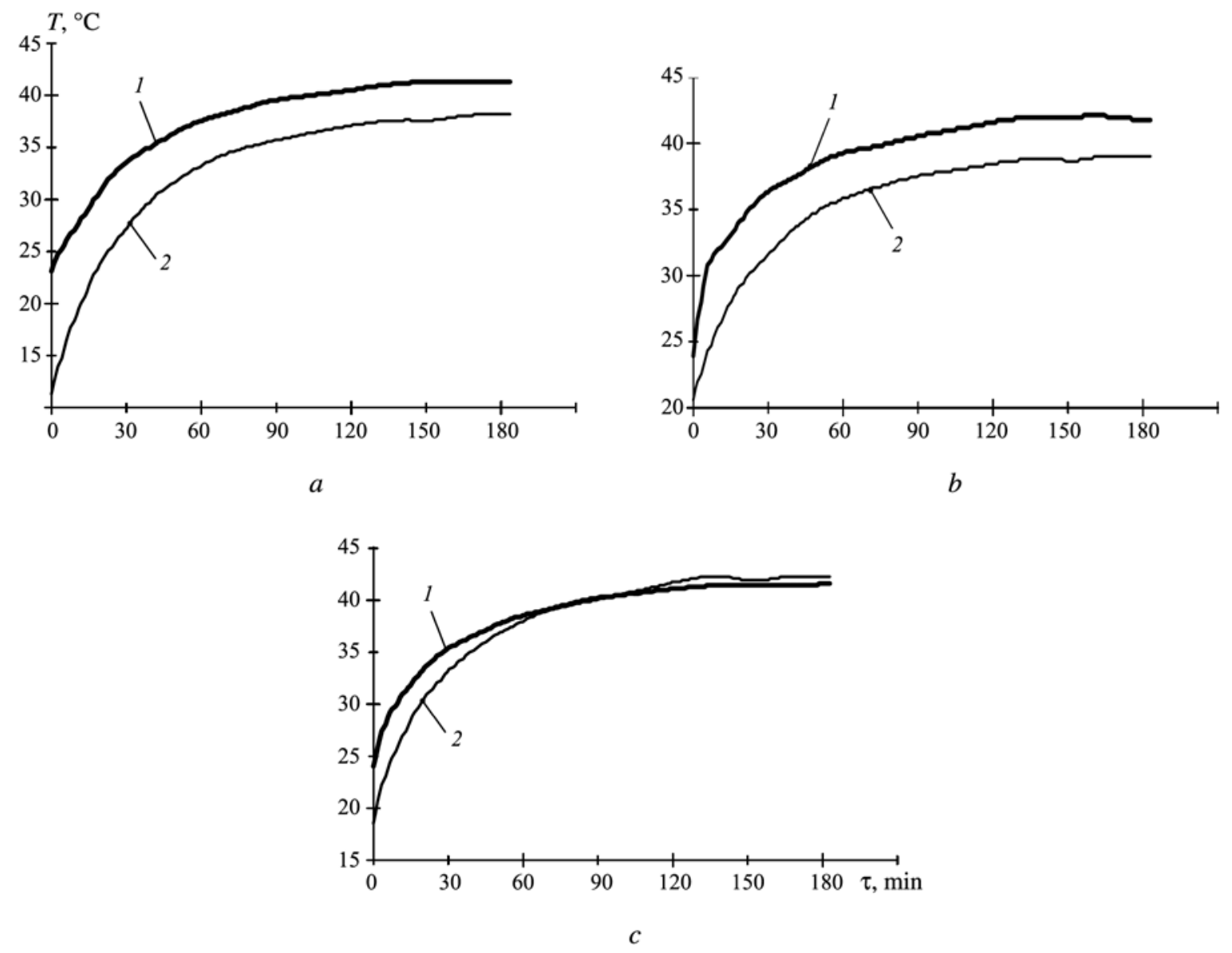

Fig. 3. Dependence of oil temperature $T$ on test time $\tau$ of gears cut by non-standard ( 1 ) and standard (2) tools in gear box (a), in support of driving shaft (b), and in support of driven shaft $(c)$.

$$
\alpha_{0}=\frac{\pi}{2}-\frac{1}{\varphi}+\frac{\pi}{2 \varphi^{2}}-\frac{3.1340681}{\varphi^{3}}+\frac{7.0173771}{\varphi^{4}}-\frac{16.824341}{\varphi^{5}}
$$

when $\varphi>5$, where $\varphi=$ inv $\alpha$ is the initial value, and $\zeta=\varphi-2.4230539$ is an auxiliary coefficient.

For $\alpha_{0}$ determined by the method of successive approximations, $\alpha$ is calculated from the formula

where $i=1,2,3, \ldots, n$.

$$
\alpha_{i}=\alpha_{i-1}+\frac{\varphi-\operatorname{inv} \alpha_{i-1}}{\tan ^{2} \alpha_{i-1}}
$$

If the inequality

$$
\left|\varphi-\operatorname{inv}_{i}\right|<10^{-10} \mathrm{rad}
$$

is satisfied, $\alpha$ is assumed to approximate $\alpha_{i}$.

In calculating $\alpha$ from these formulas, two-six approximations are required to satisfy the inequality, depending on the numerical value of $\varphi[6]$. 


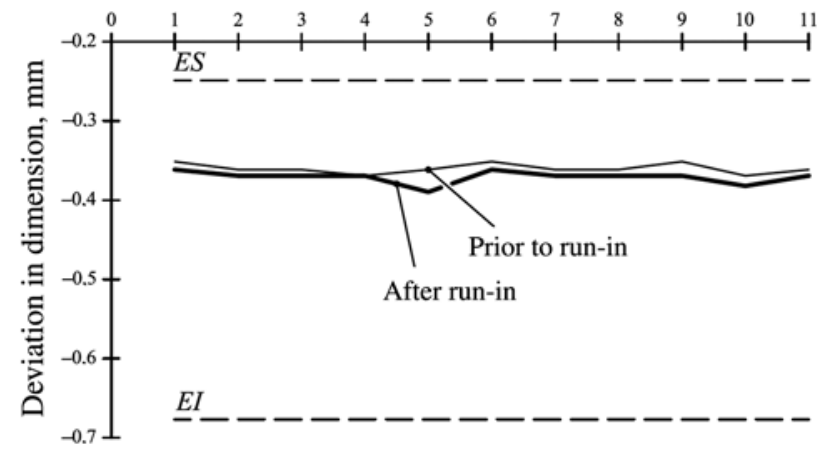

$a$

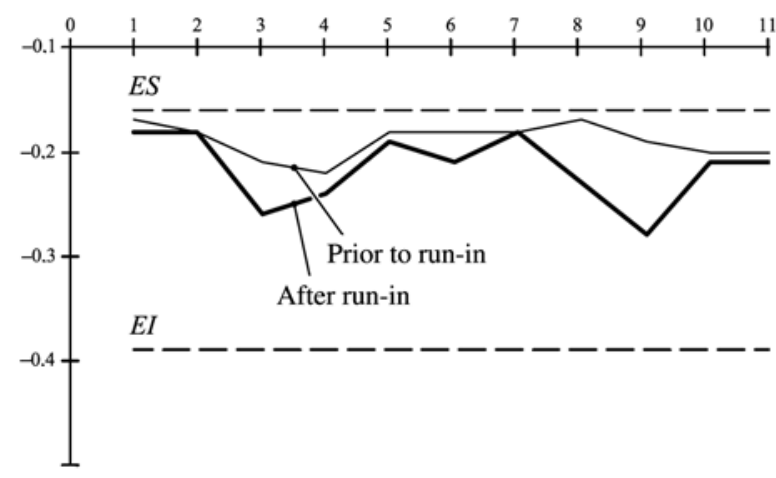

$c$
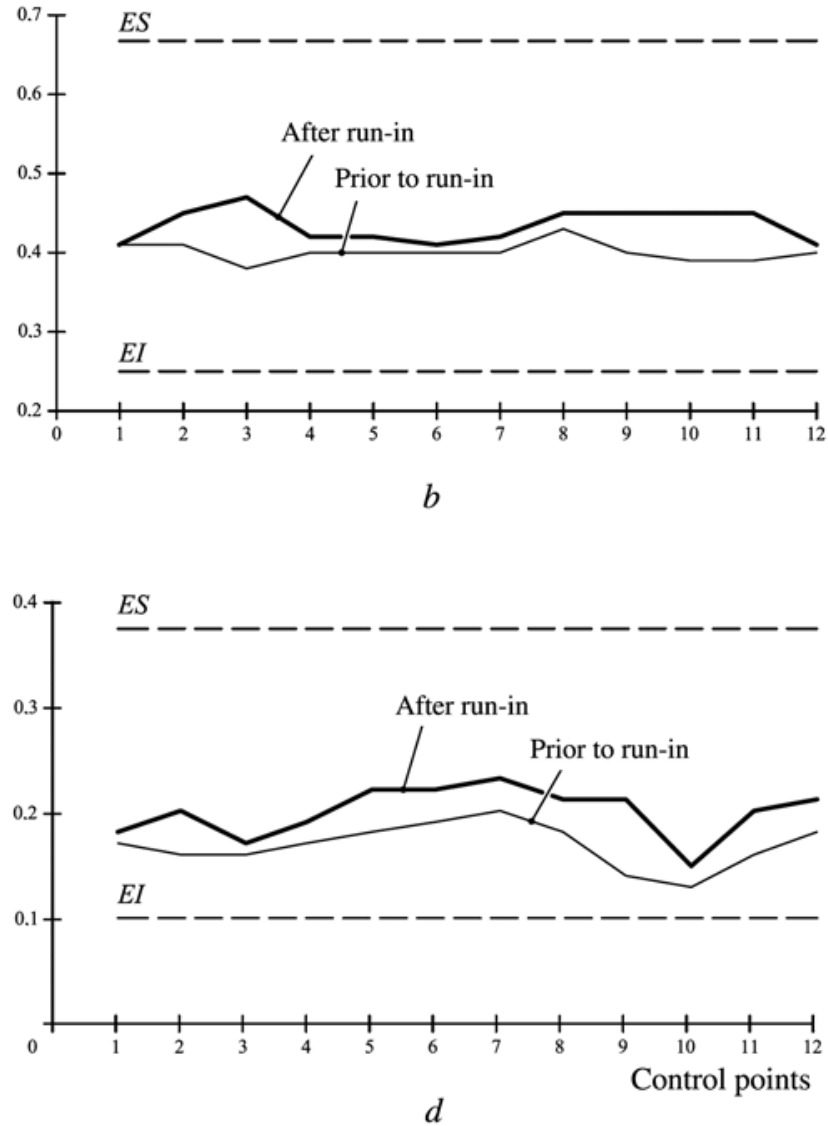

Fig. 4. Measurements of dimension with respect to rollers for gears cut by non-standard $(a, b)$ and standard $(c, d)$ tool, and for pinions $(a, c)$ and gears $(b, d): E S$ and $E I$ - upper and lower boundaries of dimensional-tolerance field.

In calculating the geometry of the gearing, therefore, values of the inverse-involute function can be determined with the accuracy required for analysis without multiple recalculation of values obtained from the basic formulas of the procedure outlined in [3].

The following indicators were examined as criteria for evaluation of the serviceability of the experimental gearing: the temperature of the oil in the gear box; the temperature of the support bearings for the driving shaft; the temperature of the support bearings for the driven shaft; and, the dimension of each gear with respect to the rollers prior to and after the run-in (for evaluation of tooth wear).

It is apparent from the plots in Fig. 3 that the temperatures of the bearings, and also the oil within the gear box differ negligibly when gears, the tooth geometry of which is calculated by different methods, are used.

Dimensional measurements with respect to the rollers were carried out prior to and after run-in for each gear at 11 points for the pinions (every seventh tooth space), and at 13 points for the gears (every sixth tooth space).

It is apparent from the plots in Fig. 4 that during the run-in, negligible work-in of the teeth occurred, as a result of which the dimension with respect to the rollers varied for each gear (decreased for the pinion, and increased for the gear). The dimension with respect to the rollers remained within tolerance limits after the run-in.

Thus, use of the method proposed for geometric analysis of gears with teeth of reduced height provides for the possibility of cutting the teeth with a standard tool. A gear, the teeth of which are cut by a standard tool are no less serviceable than a gear for which the teeth are cut by a special tool, and their use is more economically expedient, since a special tool is precluded for their machining. 


\section{REFERENCES}

1. I. A. Fainerman, "Differential reduction gear for scroll centrifuges," Vestn. Mashinostr., No. 2, 10-15 (1964).

2. V. N. Kudryavtsev, Planetary Gears [in Russian], 2nd edition, revisions and additions, Mashinostroenie, Moscow-Leningrad (1966).

3. GOST 19274-73, Internally Meshed Cylindrical Evolute Gears. Geometric Analysis, effect. Jan. 1, 1975, Izd. Standartov, Moscow (1974).

4. A. Ya. Krasilnikov and K. Yu. Muravyov, "Characteristics of geometric analysis of evolute gears with full engagement," Vestn. Mashinostr., No. 2, 15-20 (2006).

5. K. Yu. Muravyov, "Characteristics of analysis of geometric parameters of planetary gears for reduction gears in scroll centrifuges," Khim. Neftegaz. Mashinostr., No. 6, 19-23 (2009).

6. A. Ya. Krasilnikov and K. Yu. Muravyov, "Calculation of inverse-involute function," Vestn. Mashinostr., No. 4, 87-88 (2004). 